\title{
Critical point of electrolyte mixtures
}

\author{
Antti-Pekka Hynninen and Marjolein Dijkstra \\ Soft Condensed Matter Group, Debye Institute, Utrecht University, Princetonplein 5, 3584 CC Utrecht, \\ The Netherlands
}

Athanassios Z. Panagiotopoulos ${ }^{\text {a) }}$

Department of Chemical Engineering, Princeton University, Princeton, New Jersey 08544

(Received 10 May 2005; accepted 1 June 2005; published online 1 September 2005)

\begin{abstract}
The critical behavior of electrolyte mixtures was studied using grand canonical Monte Carlo simulations. Mixtures consist of large multivalent macroions and small monovalent co- and counterions. The system can be viewed as a binary mixture of macroions (with their counterions) and salt (co- and counterion pair). The primitive model description was used, in which the ions are point charges with a hard core and the solvent is treated as a uniform dielectric continuum. The grand canonical simulations are based on insertions and removals of neutral molecules: macroion with its counterions or coions and a counterion. We propose a distance biasing method that enables direct grand canonical simulations up to charge asymmetry of 10:1. We calculated the critical loci that connect the salt-free state, which consists of only macroions and counterions, with the pure salt state using mixed-field finite-size scaling with no pressure mixing. The critical parameters are determined for macroion to counterion charge asymmetries of 2:1, 3:1, and 10:1. Our results suggest that binary electrolyte mixtures are type-I mixtures, where the two components mix continuously. () 2005 American Institute of Physics. [DOI: 10.1063/1.1979490]
\end{abstract}

\section{INTRODUCTION}

In the recent years, the gas-liquid phase separation of ionic fluids has been the subject of many experimental, ${ }^{1,2}$ theoretical, ${ }^{3-8}$ and simulation studies. ${ }^{9-13}$ The simplest and most frequently used model for electrolytes is the primitive model, where the ions are point charges with a hard core and the solvent is taken into account as a uniform dielectric continuum. Panagiotopoulos and co-workers ${ }^{9,10,12}$ and Yan and de Pablo ${ }^{14-16}$ have determined the critical parameters for size- and charge-asymmetric primitive model electrolytes using grand canonical Monte Carlo (GCMC) simulations. A different approach to the problem was taken by Reščic and Linse, ${ }^{17}$ who estimated the critical parameters for 10:1 charge-asymmetric electrolyte using thermodynamic scaling Monte Carlo.

Most theoretical studies on electrolytes have considered salt-free systems with macroions and counterions, but no coions. ${ }^{18-22}$ However, in many real micellar and colloidal systems, salt is always present and often difficult to get rid of. In this paper, we consider electrolyte mixtures that consist of large multivalent macroions and small monovalent co- and counterions (salt). One can view the system as a binary mixture of macroions with their counterions and salt. Our objective is to calculate the critical loci connecting the two extremes: (i) salt-free state with macroions and counterions, and (ii) pure salt state with co- and counterions. The simulations are done for macroion to counterion charge asymmetries of 2:1, 3:1, and 10:1, using the fine-lattice primitive model GCMC method of references. ${ }^{9,10}$

Our GCMC simulations are based on insertions and removals of electroneutral sets of molecules: a macroion with

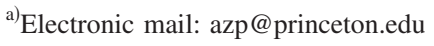

its counterions or a coions and a counterion. The main practical problem in performing the insertion and removal moves is how to choose molecule configurations that have a high probability. This problem is made especially difficult by the fact that the critical point of electrolyte systems is at low reduced temperatures, where the unlike-ion coupling is so high, that the counterions condense on the macroions. At these conditions, the standard grand canonical scheme, where the counterion positions are chosen uniformly, has low probability of acceptance. A popular solution is to use a distance biasing scheme, where counterion positions close to the macroion are favored. ${ }^{23}$ In previous studies, ${ }^{9,10}$ a distance biasing scheme has been used to calculate critical parameters up to charge asymmetry of $3: 1$.

Highly charge-asymmetric electrolytes (above 3:1) are particularly demanding to simulate with GCMC methods, because of the high number of counterions in neutral sets of molecules. Each counterion, if not at an energetically favorable position, tends to lower the acceptance of the insertion and removal steps. In order to increase acceptance at high charge asymmetries, Cheong and Panagiotopoulos ${ }^{12}$ used a reservoir GCMC method, where molecules are picked from a reservoir held at the same temperature as the GCMC simulation. This way, they were able to try insertions of highly probable molecule configurations. Consequently, they were able to determine critical parameters up to charge asymmetry of 10:1. In order to obey detailed balance in the removal step of the reservoir method, each ion is assigned to a macroion with the Stillinger-Lovett pairing protocol. ${ }^{12,24}$ The pairing protocol introduces an extra parameter $R_{c}$, the maximum allowed distance between a macroion and its counterions. As was noted in Ref. 12, the critical parameters depend (although rather weakly) on $R_{c}$. 
Our distance biasing scheme is similar to the ones used in previous works. ${ }^{9,10,23}$ By changing the biasing function such that it takes the macroion valency into account, we are able to simulate 10:1 electrolytes directly. This paper is organized as follows. In Sec. II we describe the model and the methods used in the simulations, in Sec. III we present the results, and in Sec. IV we present the conclusions from our study.

\section{MODEL AND METHODS}

We consider a system of $N_{M}$ macroions with diameter $\sigma_{M}$ carrying charge $+Q q$, and $N_{S}$ co- and $N_{-}=Q N_{M}+N_{S}$ counterions with diameter $\sigma_{I}$ carrying charges $+q$ and $-q$, respectively, where $q$ is a unit of charge. The particles interact via Coulomb potential plus hard-core repulsion such that

$$
U_{i j}\left(r_{i j}\right)= \begin{cases}\frac{q_{i} q_{j}}{D r_{i j}} & \text { for } r_{i j} \geqslant \frac{1}{2}\left(\sigma_{i}+\sigma_{j}\right) \\ +\infty & \text { for } r_{i j}<\frac{1}{2}\left(\sigma_{i}+\sigma_{j}\right)\end{cases}
$$

where $D$ is the dielectric constant of the solvent, $q_{i}$ and $q_{j}$ are the charges of ions $i$ and $j$, and $r_{i j}$ is the distance between the ions. The size asymmetry between a macro- and microion is described by

$$
\delta=\left(\frac{\sigma_{M}}{\sigma_{M I}}\right)-1=1-\frac{\sigma_{I}}{\sigma_{M I}},
$$

where $\sigma_{M I}=\frac{1}{2}\left(\sigma_{M}+\sigma_{I}\right)$ is the macroion-microion collision diameter.

The Monte Carlo simulations are performed in the grand canonical ensemble, where the thermodynamic state of the system is defined by temperature $T$, volume $V$, and chemical potentials of the macro- and coions, $\mu$ and $\mu_{S}$, respectively. In the grand canonical ensemble, the numbers of macroions $N_{M}$ and coions $N_{S}$ fluctuate. We use a cubic box of length $L$ with periodic boundary conditions. We work in reduced units with a reduced temperature

$$
T^{*}=\frac{k_{B} T D \sigma_{I}}{q^{2}} .
$$

We use the microion diameter $\sigma_{I}$ to define the reduced temperature $T^{*}$ because we wish to keep the temperature independent of the size of the macroion. The reduced density is defined as the total volume fraction of the macro- and microions, and reads

$$
\phi=\frac{\pi}{6 L^{3}}\left[\sigma_{M}^{3} N_{M}+\sigma_{I}^{3}\left(Q N_{M}+2 N_{S}\right)\right] .
$$

Chemical potentials of the macroions $\mu^{*}$ and the coions $\mu_{S}^{*}$ are defined such that at the ideal-gas limit,

$$
\left.\begin{array}{c}
\frac{\mu^{*}}{Q+1} \rightarrow T^{*} \ln \frac{N_{M} \sigma_{M I}^{3}}{V} \\
\frac{\mu_{S}^{*}}{2} \rightarrow T^{*} \ln \frac{N_{S} \sigma_{M I}^{3}}{V}
\end{array}\right\} \text { for } T^{*} \rightarrow \infty, \quad \phi \rightarrow 0
$$

The reduced pressure is defined in units of the microion diameter $\sigma_{I}$ as

$$
P^{*}=\frac{P \sigma_{I}^{3}}{k_{B} T} .
$$

We again use the microion diameter $\sigma_{I}$ to define $P^{*}$ in order to keep it independent of the macroion size.

Long-range Coulombic interactions are calculated using the Ewald summation method ${ }^{25,26}$ with conducting boundary conditions, 518 Fourier-space vectors, and real-space damping parameter $\kappa=5$. In order to speed up simulations, we use the fine-lattice discretization method of Panagiotopoulos and Kumar. $^{27}$ The advantage of the lattice method is that it allows us to precalculate all the Coulombic interactions in the beginning of the simulation. During the simulation, the pair interactions are determined by a simple table lookup. Denoting the lattice spacing by $a$, we can define a lattice refinement parameter

$$
\xi=\sigma_{M I} / a
$$

Continuum is recovered when $\xi \rightarrow \infty$. The presence of salt introduces an additional length scale, the microion diameter $\sigma_{I}$, and, therefore, we introduce a second lattice refinement parameter

$$
\xi_{I}=\sigma_{I} / a=(1-\delta) \xi
$$

The effect of lattice discretization on the critical behavior of 1:1 systems was studied in Ref. 27, where it was found that the normal vapor-liquid phase coexistence is recovered for $\xi \geqslant 3$. For $\xi \leqslant 2$, no vapor-liquid phase coexistence is possible, but instead the system phase separates into a disordered and an antiferromagnetic phase. In order to ensure that the salt in our system has a vapor-liquid phase coexistence, we use microion refinement $\xi_{I} \geqslant 3$. For the macroions, we use $\xi \geqslant 10$, as it is known from an earlier work ${ }^{28}$ that the difference between the critical parameters at $\xi=10$ and $\xi$ $\rightarrow \infty$ is small, about $1 \%$ for the temperature and about $4 \%$ for the density.

Our GCMC simulations are based on insertions and removals of two types of neutral molecules: (i) a macroion with $Q$ counterions and (ii) a coions with a counterion. As the vapor-liquid phase separation occurs at low reduced temperatures, the counterions have a strong tendency to form clusters around macroions and coions. Therefore, purely random (i.e., uniform) insertions and removals of counterions lead to very low acceptance rates. In order to improve the acceptance, we use a distance biasing scheme. In the distance biasing scheme, counterions are inserted with a biased probability distribution that increases the probability of inserting a counterion close to a macroion or a coions. In the present case, a natural choice for the biased probability distribution is

$$
w_{\mathrm{bias}}\left(r_{i j}\right)= \begin{cases}\exp \left(\frac{Q_{b}}{T^{*}} \frac{1}{r_{i j}}\right) & \text { for } r_{i j} \geqslant \frac{1}{2}\left(\sigma_{i}+\sigma_{j}\right) \\ 0 & \text { for } r_{i j}<\frac{1}{2}\left(\sigma_{i}+\sigma_{j}\right),\end{cases}
$$

that is the Boltzmann factor of a Coulomb interaction between a positive ion of charge $Q_{b}$ and a counterion. Note that the probability distribution in Eq. (9) is not normalized. 
In Eq. (9), the "bias charge" $Q_{b}$ is a free parameter that can be adjusted to obtain maximum acceptance. We use, $Q_{b}=Q$ for molecules with $Q=1$ and 3 , and $Q_{b}=6$ for molecules with $Q=10$. As we observed, for $Q \approx 10$, it is favorable to choose $Q_{b}<Q$, in order to account for counterioncounterion repulsion that reduces the effective charge seen by the counterions. For $Q=10$, optimal $Q_{b}$ was determined by running a short simulation with different $Q_{b}$ and choosing the one that gave the highest acceptance. The choice $Q_{b}=6$ was also confirmed by fitting the effective macroionmacroion pair potential (obtained from the radial distribution function at low density) with $Q_{b} / r$. For $Q=3$ and 1 , we did not try to find an optimal $Q_{b}$ because $Q_{b}=Q$ already gave high acceptance. Note that $Q_{b}=1$ was used for all $Q$ in previous works. ${ }^{9,10}$ This gives relatively low acceptance for $Q$ $>1$, although still much higher than without biasing at all.

In the following, we describe in detail the insertion and the removal steps of a macroion; the insertion and removal of a coions are completely analogous. In the insertion step, the trial position of the macroion is chosen uniformly. Counterion positions are chosen according to the probability distribution $w_{\text {bias }}\left(R_{k_{x} k_{y} k_{z}}\right)$ given by Eq. (9), where $R_{k_{x} k_{y} k_{z}}$ is the distance between a macroion center and a lattice site $\left(k_{x}, k_{y}, k_{z}\right)$. In practice, the cumulative probability

$$
W_{k_{x} k_{y} k_{z}}=\frac{1}{Z} \sum_{\ell_{z}=1}^{k_{z}} \sum_{\ell_{y}=1}^{k_{y}} \sum_{\ell_{x}=1}^{k_{x}} w_{\text {bias }}\left(R_{\ell_{x} \ell_{y} \ell_{z}}\right)
$$

is precalculated into a three-dimensional $\xi L \times \xi L \times \xi L$ matrix at the beginning of a simulation. In Eq. (10),

$$
Z=\sum_{\ell_{z}=1}^{\xi L} \sum_{\ell_{y}=1}^{\xi L} \sum_{\ell_{x}=1}^{\xi L} w_{\text {bias }}\left(R_{\ell_{x} \ell_{y} \ell_{z}}\right)
$$

is the appropriate normalization factor, i.e., sum of $w_{\text {bias }}$ over all lattice positions $[1, \ldots, \xi L ; 1, \ldots, \xi L ; 1, \ldots, \xi L]$. In the simulation, we find the lattice site $\left(k_{x}, k_{y}, k_{z}\right)$ for the trial position of the counterions by going through the matrix and finding the first lattice site $\left(k_{x}, k_{y}, k_{z}\right)$, for which the cumulative probability $W_{k_{x} k_{y} k_{z}}$ is smaller than a random number drawn from a uniform $[0,1]$ distribution. We use a binary search algorithm to make the search efficient. This procedure is repeated for each of the $Q$ counterions. Once all the counterion lattice sites are found, the insertion is accepted according to the criteria

$$
\begin{aligned}
\operatorname{acc}(N \rightarrow N+1) & \\
=\min & {\left[1,\left(\frac{V}{N+1}\right)^{Q+1} \frac{B_{\text {ins }}}{B_{\text {del }}} \exp \left[\left(\mu^{*}-U_{N+1}+U_{N}\right) / T^{*}\right]\right], }
\end{aligned}
$$

where $U_{N}$ and $U_{N+1}$ are the potential energies (in units of $k_{B} T$ ) before and after insertion, and

$$
\frac{B_{\mathrm{ins}}}{B_{\mathrm{del}}}=\left(\frac{Z}{\xi^{3} L^{3}}\right)^{Q} \prod_{i=1}^{Q} \frac{N_{-}-(i-1)}{\sum_{j=1, j \neq\left[k_{1}, \ldots, k_{i-1}\right]}^{N_{-}} \exp \left(Q_{b} / T^{*} r_{j}\right)}
$$

is a factor that corrects for the bias, and hence detailed balance is satisfied.
In the removal step, a macroion is chosen randomly and the first counterion is chosen according to the probability

$$
\frac{\exp \left(Q_{b} / T^{*} r_{k_{1}}\right)}{\sum_{j=1}^{N_{-}} \exp \left(Q_{b} / T^{*} r_{j}\right)}
$$

When choosing the second counterion, the first $\left(k_{1}\right)$ counterion has to be removed from the normalization. Thus, the second counterion is chosen according to the probability

$$
\frac{\exp \left(Q_{b} / T^{*} r_{k_{2}}\right)}{\sum_{j=1, j \neq k_{1}}^{N_{-}} \exp \left(Q_{b} / T^{*} r_{j}\right)} \text {. }
$$

Continuing in this manner, we have for the $Q$ th counterion

$$
\frac{\exp \left(Q_{b} / T^{*} r_{k_{Q}}\right)}{\sum_{j=1, j \neq\left[k_{1}, \ldots, k_{Q-1}\right]}^{N_{-}} \exp \left(Q_{b} / T^{*} r_{j}\right)} .
$$

When all the $Q$ counterions are chosen, the removal step is accepted according to the criteria

$$
\begin{aligned}
& \operatorname{acc}(N+1 \rightarrow N) \\
& =\min \left[1,\left(\frac{N+1}{V}\right)^{Q+1} \frac{B_{\mathrm{del}}}{B_{\text {ins }}} \exp \left[\left(-\mu^{*}+U_{N+1}-U_{N}\right) / T^{*}\right]\right] .
\end{aligned}
$$

In practice, when choosing the first counterion, we calculate the probability in Eq. (14) for every counterion, and then, as in the case of insertion, use a binary search for the cumulative probability. For the second counterion, we simply exclude the first counterion from the cumulative sum and repeat the search.

In addition to the insertion and removal steps, displacement moves are carried out for microions. We noticed that displacement moves help the system to equilibrate. The portion of displacement moves over all the moves was set to $10 \%$.

Simulations consist of equilibration and sampling runs. In a sampling run, a histogram containing the number of macroions $N_{M}$, coions $N_{S}$, and total energy $U$ is collected. In order to determine the critical point, a simulation is done at a temperature that is close but above the critical temperature. Close to the critical point, systems sample a broad density range, due to critical fluctuations. From the $\left(N_{M}, N_{S}, U\right)$ histogram, the critical point is determined using a mixed-field finite-scaling method ${ }^{29}$ that assumes Ising criticality with no pressure mixing. The effects of pressure mixing on the critical parameters, if any, are expected to be minor, but require substantial additional computational effort. ${ }^{30}$ Typically, a single simulation run is not enough to determine the critical point to a desired accuracy, and therefore multiple runs with different macroion chemical potential $\mu^{*}$ are combined using a histogram reweighting technique. ${ }^{31,32}$

The pressure is given by the relation

$$
\frac{P^{*} V}{\sigma_{I}^{3}}=\frac{P V}{k_{B} T}=\ln \Xi\left(\mu, \mu_{S}, V, T\right)+\text { const, }
$$

where $\Xi\left(\mu, \mu_{S}, V, T\right)$ is the grand canonical partition function, whose value can be calculated from the histogram data. 
TABLE I. Critical parameters for system (a) with charge asymmetry of 2:1 and size asymmetry $\delta=0.5$. The parameters are for box size $L=12 \sigma_{M I}$. The numbers in parentheses refer to the statistical uncertainty of the last decimal place shown.

\begin{tabular}{llllll}
\hline \hline$\mu_{S}^{*}$ & $\mu_{c}^{*}$ & $x_{S}$ & $T_{c}^{*} \times 10^{2}$ & $\phi_{c} \times 10^{2}$ & $P_{c}^{*} \times 10^{3}$ \\
\hline$-\infty$ & $-2.0260(2)$ & $0(0)$ & $4.770(5)$ & $5.7(1)$ & $0.328(4)$ \\
$-\infty$ & $-2.0254(4)$ & $0(0)$ & $4.75(1)$ & $5.8(1)^{\mathrm{a}}$ & $\ldots$ \\
$-\infty$ & $-2.02557(9)$ & $0(0)$ & $4.752(3)$ & $5.99(6)$ & $0.352(4)^{\mathrm{b}}$ \\
-1.500 & $-2.0315(4)$ & $0.0496(1)$ & $4.798(8)$ & $5.5(1)$ & $0.308(3)$ \\
-1.500 & $-2.0300(6)$ & $0.0503(6)$ & $4.75(2)$ & $5.91(8)$ & $0.325(2)^{\mathrm{b}}$ \\
-1.300 & $-2.0684(6)$ & $0.35320(2)$ & $4.733(8)$ & $5.5(1)$ & $0.341(6)$ \\
-1.250 & $-2.1049(6)$ & $0.531(2)$ & $4.708(8)$ & $5.4(2)$ & $0.45(1)$ \\
-1.200 & $-2.1944(5)$ & $0.760(1)$ & $4.70(1)$ & $5.3(2)$ & $0.74(3)$ \\
-1.175 & $-2.3115(1)$ & $0.8868(5)$ & $4.817(8)$ & $5.1(2)$ & $1.20(2)$ \\
\hline
\end{tabular}

${ }^{\mathrm{a}}$ For box size $L=15 \sigma_{M I}$, from Ref. 10 .

${ }^{\mathrm{b}}$ For box size $L=15 \sigma_{M I}$.

In the limit of low density, where the system behaves as an ideal gas, a plot of $\ln \Xi$ vs $\left(N_{M}+N_{S}\right)$ gives a straight line with unit slope. The "const" in Eq. (18) is given by the extrapolation of this line to the limit $N_{M}+N_{S} \rightarrow 0$.

\section{RESULTS}

We study four different systems with charge and size asymmetries of (a) $2: 1$ and $\delta=0.5$, (b) $3: 1$ and $\delta=0.667$, (c) 10:1 and $\delta=0.9$, and (d) $10: 1$ and $\delta=0.667$. For systems (a)-(c), the size asymmetry was chosen so that a macroioncounterion pair and a coions-counterion pair have equal Coulomb potentials at contact. The lattice refinement parameter $\xi$ is set to $\xi=10$ in systems (a), (b), and (d). According to Eq. (8), this gives $\xi_{I}=5$ in system (a) and $\xi_{I}=3.33$ in systems (b) and (d). In system (c), $\xi=10$ would give a microion lattice refinement of $\xi_{I}=1$. As was mentioned in Sec. II, only systems with $\xi_{I} \geqslant 3$ have a vapor-liquid phase separation. Therefore, in system (c), we use a finer lattice with $\xi=30$, to make sure that the salt has a vapor-liquid phase separation $\left(\xi_{I}=3\right)$.

In our simulations, we start from a salt-free state with $\mu_{S}^{*}=-\infty$ and add salt by increasing $\mu_{S}^{*}$ in steps. For each step, the critical parameters are determined. We characterize the amount of the added salt by the salt mole fraction, defined as

$$
x_{S}=\frac{N_{S}}{N_{M}+N_{S}} .
$$

With this definition, $x_{S}=0$ corresponds to a salt-free state and $x_{S}=1$ corresponds to a pure salt state. Our results are listed in Tables I-IV. Unless otherwise indicated, the parameters are for box size $L=12 \sigma_{M I}$. Error estimates are calculated from (two to four) independent runs at different temperatures using the Student's $t$-test with $90 \%$ confidence interval.

Figures $1-4$ show the critical temperature $T_{c}^{*}$ as a function of the salt mole fraction $x_{S}$. The pure salt critical temperature is taken from Ref. 27; we use $\xi=5$ results for system (a), whose ions are on a lattice with $\xi_{I}=5$, and $\xi=3$ results for systems (b)-(d), whose ions are on a lattice with $\xi_{I}$ =3.33. As can be seen from Figs. 1-3, in systems (a)-(c), where the pure salt has a higher critical temperature than the salt-free state, $T_{c}^{*}\left(x_{S}\right)$ is not monotonic, but goes through a minimum (at $x_{S} \approx 0.6-0.8$ ). In system (c), we did not reach high enough salt fractions to locate the minimum explicitly, but as the inset in Fig. 3 shows, $T_{c}^{*}$ is decreasing with increasing $x_{S}$. Therefore, we expect there to be a minimum. We have extrapolated lines from our data to pure salt to indicate the expected qualitative behavior of $T_{c}^{*}$ over the complete composition range. This extrapolation is, of course, only approximate-it would take significantly more computa-

TABLE II. Critical parameters for system (b) with charge asymmetry of 3:1 and size asymmetry $\delta=0.667$. The parameters are for box size $L=12 \sigma_{M I}$. The numbers in parentheses refer to the statistical uncertainty of the last decimal place shown.

\begin{tabular}{llclll}
\hline \hline$\mu_{S}^{*}$ & $\mu_{c}^{*}$ & $x_{S}$ & $T_{c}^{*} \times 10^{2}$ & $\phi_{c} \times 10^{2}$ & $P_{c}^{*} \times 10^{3}$ \\
\hline$-\infty$ & $-2.6672(2)$ & $0(0)$ & $4.488(5)$ & $7.3(2)$ & $0.095(4)$ \\
-1.17 & $-2.7077(9)$ & $0.3710(6)$ & $4.40(2)$ & $7.5(2)$ & $0.120(6)$ \\
-1.13 & $-2.7282(4)$ & $0.4824(9)$ & $4.354(5)$ & $7.5(4)$ & $0.146(3)$ \\
-1.12 & $-2.744(1)$ & $0.5446(9)$ & $4.33(3)$ & $7.3(2)$ & $0.164(7)$ \\
-1.07 & $-2.8294(3)$ & $0.749(2)$ & $4.26(1)$ & $7.3(2)$ & $0.28(1)$ \\
-1.07 & $-2.8305(5)$ & $0.752(2)$ & $4.23(1)$ & $7.41(9)$ & $0.33(2)^{\mathrm{a}}$ \\
-1.05 & $-2.8855(3)$ & $0.8169(6)$ & $4.270(4)$ & $7.7(1)$ & $0.410(1)$ \\
-1.03 & $-2.9693(6)$ & $0.8818(6)$ & $4.320(2)$ & $7.2(2)$ & $0.590(2)$ \\
-1.02 & $-3.039(1)$ & $0.9157(8)$ & $4.382(4)$ & $6.9(3)$ & $0.77(1)$ \\
-1.01 & $-3.167(2)$ & $0.9535(3)$ & $4.505(0)$ & $6.5(4)$ & $1.0(2)$ \\
-1.00 & $-3.32(1)$ & $0.977(2)$ & $4.62(1)$ & $5.6(5)$ & $1.4(1)$ \\
\hline \hline
\end{tabular}

${ }^{\mathrm{a}}$ For box size $L=15 \sigma_{M I}$. 
TABLE III. Critical parameters for system (c) with charge asymmetry of 10:1 and size asymmetry $\delta=0.9$. The parameters are for box size $L=12 \sigma_{M I}$. The numbers in parentheses refer to the statistical uncertainty of the last decimal place shown.

\begin{tabular}{lccccl}
\hline \hline$\mu_{S}^{*}$ & $\mu_{c}^{*}$ & $x_{S}$ & $T_{c}^{*} \times 10^{2}$ & $\phi_{c} \times 10^{2}$ & $P_{c}^{*} \times 10^{6}$ \\
\hline$-\infty$ & $-6.801(1)$ & $0(0)$ & $3.28(1)$ & $15.2(1)$ & $2.444(2)$ \\
-1.26 & $-6.807(1)$ & $0.093(1)$ & $3.28(1)$ & $14.5(5)$ & $1.2(2)$ \\
-1.20 & $-6.818(3)$ & $0.208(4)$ & $3.28(2)$ & $14.3(8)$ & $1.3(3)$ \\
-1.10 & $-6.8784(8)$ & $0.559(7)$ & $3.26(2)$ & $15(1)$ & $1.5(2)$ \\
-1.08 & $-6.9078(1)$ & $0.641(3)$ & $3.24(1)$ & $15(2)$ & $1.7(2)$ \\
\hline \hline
\end{tabular}

tional resources than those available to us to enable calculations for systems with large amounts of salt because of the difficulty in sampling macroion insertions and removals.

In Figs. 1-3, the decrease in the critical temperature at $x_{S}<0.8$ can be explained by a more general result, which states that external disturbances, such as walls, added components, etc., lower the critical temperature. ${ }^{33}$ At $x_{S}>0.8$, the critical temperature increases to reach the pure salt critical point. In contrast, in system (d), where pure salt has a lower critical temperature than the salt-free state, $T_{c}^{*}\left(x_{S}\right)$ decreases monotonically, see Fig. 4.

In Figs. 5-8, we plot the critical volume fraction $\phi_{c}$ as a function of the salt mole fraction $x_{S}$. In systems (a)-(c), $\phi_{c}$ is more or less a constant for $0<x_{S}<0.9$, and at $x_{S}>0.9, \phi_{c}$ starts to decrease rapidly towards the pure salt critical point. In the 10:1 systems [(c) and (d)], we were not able to calculate critical parameters beyond $x_{S} \approx 0.66$ for box size $L$ $=12 \sigma_{M I}$, due to sampling problems: the acceptance of macroion insertions in these systems was below $0.1 \%$. However, we were able to calculate critical parameters up to $x_{S}=0.76$ for system (d) (10:1 and $\delta=0.667)$ using box size $L=10 \sigma_{M I}$. These results are denoted by the triangles in Fig. 8. As the statistical accuracy is poor, it is hard to make any definite conclusions. However, it is tempting to argue that $\phi_{c}\left(x_{S}\right)$ tends towards the pure salt limit as $x_{S} \rightarrow 1$.

In order to get an idea of the system size dependence of our results, we recalculated some of the critical points for different box size $L$. In system (a) $(2: 1$ and $\delta=0.5)$, the two lowest salt critical points were recalculated using $L=15 \sigma_{M I}$, see Figs. 1 and 5 and Table I. We find considerable system size dependence for this system. The is because, for box size
$L=12 \sigma_{M I}$, there are not enough data points at the low-density end of the histogram to fit the Ising curve completely. At higher salt concentration, there are more data points at the low-density part of the histograms and this system size dependence disappears. According to our histogram data, the Ising curve can be fitted completely when $x_{S} \approx 0.6$. In system (a), our results for box size $L=15 \sigma_{M I}$ agree very well with the earlier results in Ref. 10 that are also for $L=15 \sigma_{M I}$. In system (b) (3:1 and $\delta=0.667)$, the critical point at $\mu_{S}^{*}$ $=-1.07$ was recalculated using $L=15 \sigma_{M I}$, and, as can be seen from Table II, the critical parameters are almost within the error bars of the results for $L=12 \sigma_{M I}$. In system (d) (10:1 and $\delta=0.667)$, we recalculated critical points at $\mu_{S}^{*}=-1.33$ and at $\mu_{S}^{*}=-1.17$ using a box size $L=10 \sigma_{M I}$, see Table IV. At $\mu_{S}^{*}=-1.17$, all critical parameters (except $\mu_{c}^{*}$ ) are, within the error bars, indistinguishable. However, at $\mu_{S}^{*}=-1.33$, we find significant differences between the results for $L=12 \sigma_{M I}$ and for $L=10 \sigma_{M I}$.

Figure 9 shows the critical-point loci in the (temperature $T^{*}$, pressure $P^{*}$ ) plane. From two independent measurements with box sizes $L=15 \sigma_{M I}$ and $19 \sigma_{M I}$, we estimated the pure salt critical pressure $P^{*}=0.00297(4)$ using Eq. (18) and critical temperature $T^{*}=0.0528(2)$. We are not aware of previous work that reports critical pressure of electrolytes. As can be seen from Fig. 9, the pure salt state has a higher pressure than any of the salt-free states. The reason for this is the ideal-gas contribution to the pressure $\left(P_{\text {id }} V=N k_{B} T\right)$, which is high for the pure salt state because it has the highest number density of particles than any of the salt-free states (although it has the lowest volume fraction, see Figs. 5-8). Figure 9 also shows that, upon adding salt, the pressure increases very

TABLE IV. Critical parameters for system (d) with charge asymmetry of 10:1 and size asymmetry $\delta=0.667$. The parameters are for box size $L=12 \sigma_{M I}$. The numbers in parentheses refer to the statistical uncertainty of the last decimal place shown.

\begin{tabular}{lccccl}
\hline \hline$\mu_{S}^{*}$ & $\mu_{c}^{*}$ & $x_{S}$ & $T_{c}^{*} \times 10^{2}$ & $\phi_{c} \times 10^{2}$ & $P_{c}^{*} \times 10^{3}$ \\
\hline$-\infty$ & $-22.744(2)$ & $0(0)$ & $11.10(1)$ & $11.2(2)$ & $0.101(5)$ \\
-1.80 & $-22.737(6)$ & $0.126(3)$ & $10.89(3)$ & $11.8(3)$ & $0.18(1)$ \\
-1.67 & $-22.732(2)$ & $0.202(2)$ & $10.74(2)$ & $11.9(5)$ & $0.26(2)$ \\
-1.53 & $-22.726(3)$ & $0.30(1)$ & $10.49(3)$ & $13(1)$ & $0.40(4)$ \\
-1.33 & $-22.718(3)$ & $0.48(1)$ & $9.73(2)$ & $15(1)$ & $0.69(1)$ \\
-1.33 & $-22.723(3)$ & $0.50(2)$ & $9.83(2)$ & $12(1)$ & $0.521(9)^{\mathrm{a}}$ \\
-1.30 & $-22.733(1)$ & $0.535(9)$ & $9.732(5)$ & $13.4(8)$ & $0.683(5)^{\mathrm{a}}$ \\
-1.17 & $-22.758(5)$ & $0.661(7)$ & $8.61(8)$ & $16(1)$ & $1.2(1)$ \\
-1.17 & $-22.771(2)$ & $0.667(4)$ & $8.79(5)$ & $16.2(7)$ & $1.2(5)^{\mathrm{a}}$ \\
-1.1 & $-22.8229(4)$ & $0.755(2)$ & $7.99(7)$ & $15(1)$ & $1.3(1)^{\mathrm{a}}$ \\
\hline \hline
\end{tabular}

${ }^{\mathrm{a}}$ For box size $L=10 \sigma_{M I}$. 


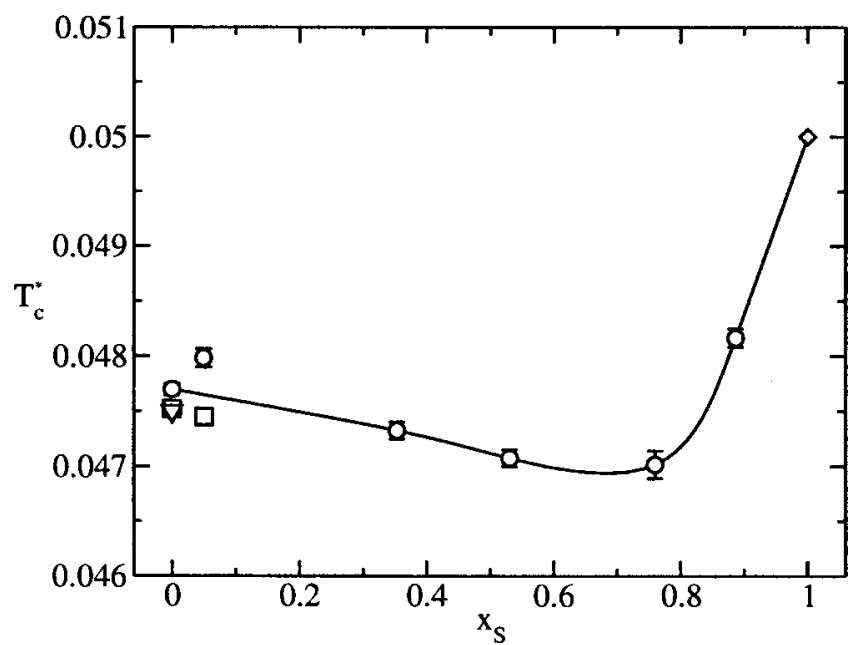

FIG. 1. Critical temperature $T_{c}^{*}$ of system (a) with charge asymmetry of $2: 1$ and size asymmetry $\delta=0.5$, as a function of the salt mole fraction $x_{S}$. The points marked with the circles $(\bigcirc)$ are for box size $L=12 \sigma_{M I}$, while those marked with the squares $(\square)$ and triangles $(\nabla)$ are for box size $L=15 \sigma_{M I}$ The line is a guide to the eye. The pure salt critical temperature at $x_{S}=1(\diamond)$ is from Ref. 27 and salt-free critical temperature at $x_{S}=0(\nabla)$ is from Ref. 10 $\left(L=15 \sigma_{M I}\right)$. The error bars smaller than the symbol size are not drawn.

gradually at first, and then in big steps at higher salt concentrations. This is why, even at the highest coions concentration $x_{S}=0.977$ [system (c)], where the system consists almost completely of salt, the pressure is well below the pure salt state. Note that the critical-point loci of system (c) (10:1 and $\delta=0.9$ ) is missing from Fig. 9. In this high size asymmetry case, all pressures are much smaller than in the other systems, see Table III. This is because the length scale in our definition of $P^{*}$ [see Eq. (10)] is the microion diameter and for high size asymmetries, the factor $\sigma_{I}^{3}$ is very small.

We can conclude that the electrolyte mixtures studied here are type-I mixtures, where the two components mix continuously. ${ }^{34}$ Clearly, this conclusion hold less firmly for system (c), where we were not able to reach high enough $x_{S}$

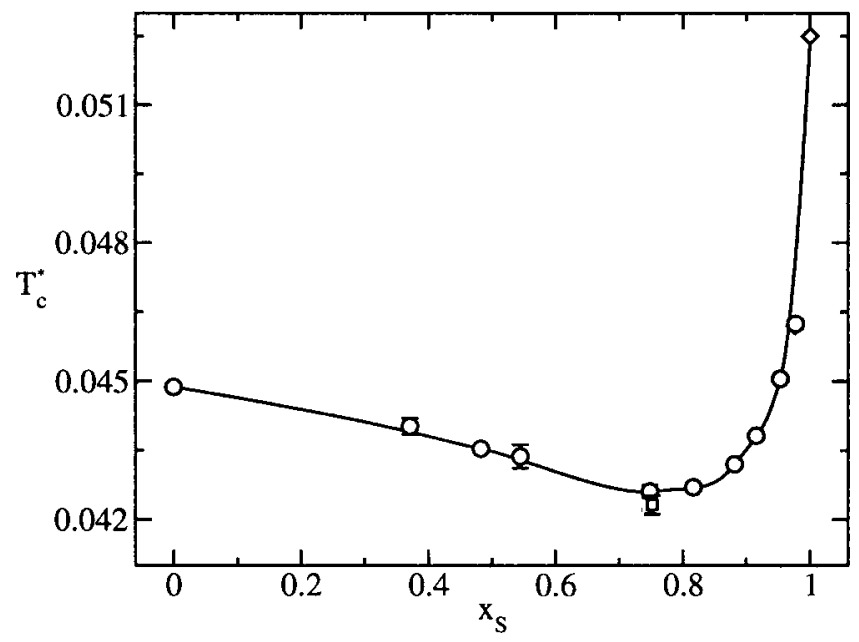

FIG. 2. Critical temperature $T_{c}^{*}$ of system (b) with charge asymmetry of $3: 1$ and size asymmetry $\delta=0.667$, as a function of the salt mole fraction $x_{S}$. The points marked with the circles $(\bigcirc)$ are for box size $L=12 \sigma_{M I}$ and the point marked with the square $(\square)$ is for box size $L=15 \sigma_{M I}$. The line is a guide to the eye. The pure salt critical temperature at $x_{S}=1(\diamond)$ is from Ref. 27. The error bars smaller than the symbol size are not drawn.

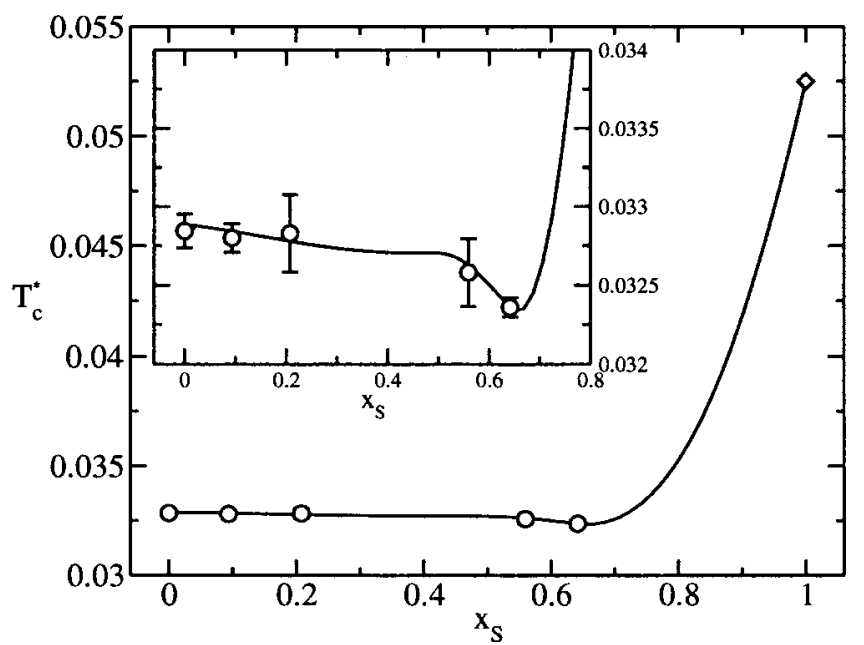

FIG. 3. Critical temperature $T_{c}^{*}$ of system (c) with charge asymmetry of 10:1 and size asymmetry $\delta=0.9$, as a function of the salt mole fraction $x_{S}(\bigcirc)$. The results are for box size $L=12 \sigma_{M I}$. The line is a guide to the eye. The pure salt critical temperature at $x_{S}=1(\diamond)$ is from Ref. 27. The error bars smaller than the symbol size are not drawn.

to see a convergence towards the pure salt limit. However, our data does not give evidence of more complex mixing behavior either.

In all systems, the macroion vapor phase is also the vapor phase for the salt, and the macroion liquid phase is also the liquid phase for the salt. In other words, when the system fluctuates between vapor and liquid phases, macroion and salt densities are coupled. We observed, that for a fixed coions chemical potential $\mu_{S}^{*}$, the ratio between macroion and salt number densities is constant to a very good approximation. The coupling between the macroion and the salt densities also explains why the errors in the salt mole fraction $x_{S}$ are so small. Figures 10 and 11 show the radial distribution functions of positive like-charged species for the 3:1 (b) and 10:1 (d) systems with $\delta=0.667$, respectively. The radial dis-

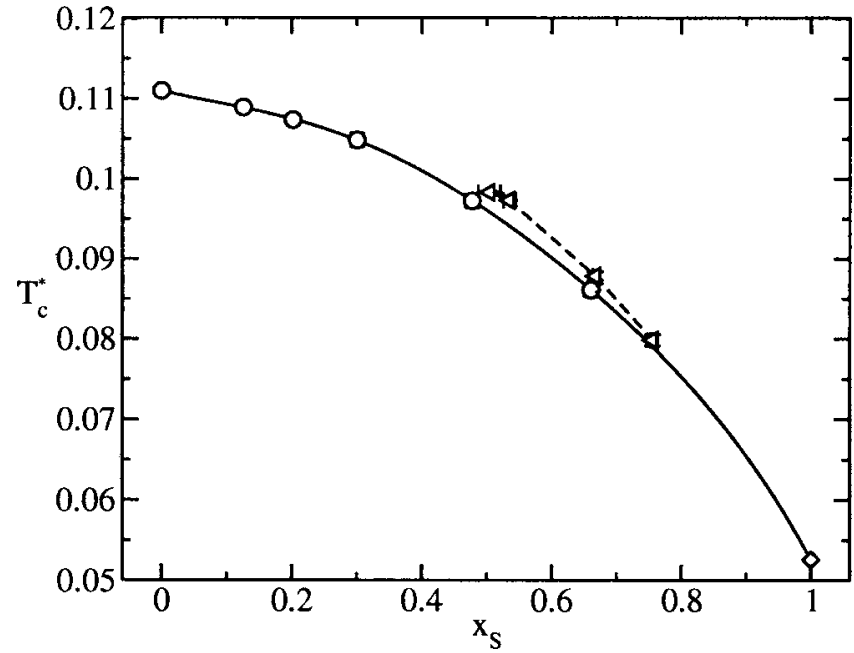

FIG. 4. Critical temperature $T_{c}^{*}$ of system (d) with charge asymmetry of 10:1 and size asymmetry $\delta=0.667$, as a function of the salt mole fraction $x_{S}$. The points marked with the circles $(\bigcirc)$ are for box size $L=12 \sigma_{M I}$ and those marked with the triangles $(\triangleleft)$ are for box size $L=10 \sigma_{M I}$. The lines are guides to the eye. The pure salt critical temperature at $x_{S}=1(\diamond)$ is from Ref. 27. The error bars smaller than the symbol size are not drawn. 


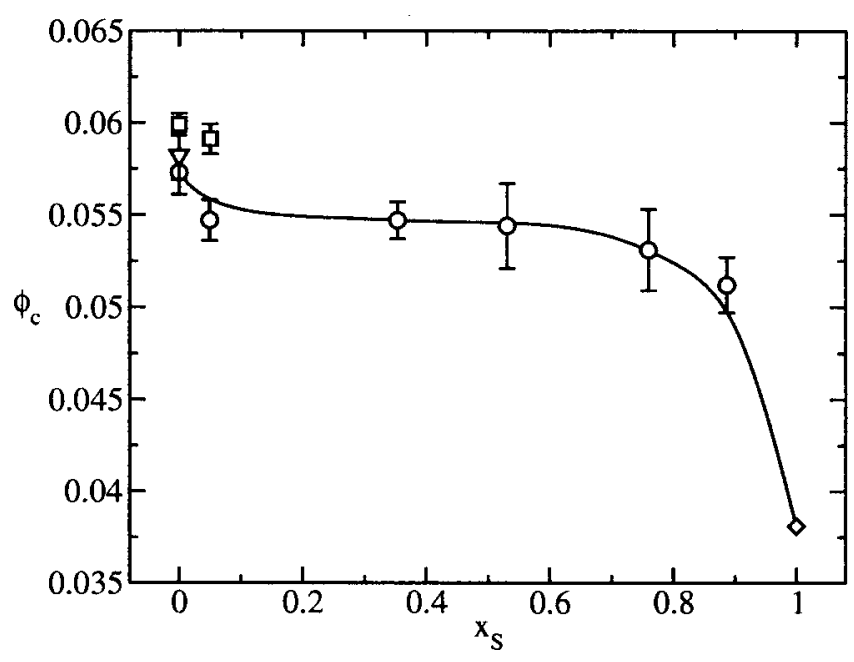

FIG. 5. Critical volume fraction $\phi_{c}\left(x_{S}\right)$ of system (a) with charge asymmetry of $2: 1$ and size asymmetry $\delta=0.5$, as a function of the salt mole fraction $x_{S}$. The points marked with the circles $(\bigcirc)$ are for box size $L=12 \sigma_{M I}$, while those marked with the squares $(\square)$ and triangles $(\nabla)$ are for box size $L$ $=15 \sigma_{M I}$. The pure salt critical temperature at $x_{S}=1(\diamond)$ is from Ref. 27 and the salt-free critical temperature at $x_{S}=0(\nabla)$ is from Ref. 10. The line is a guide to the eye.

tribution functions in Figs. 10 and 11 are calculated in the vapor phase, close to the $x_{S}=0.48$ critical point at $T^{*}$ $\approx 1.03 T_{c}^{*}$ (i.e., slightly above the critical temperature). As Figs. 10 and 11 show, all radial distributions are peaked close to their contact distance. Since, in the low-density vapor phase, the effective interaction potential is, to a good approximation, equal to negative logarithm of the radial distribution function $\left[u_{\text {eff }}=-\ln g(r)\right]$, this implies effective attraction between all like-charged species. The effective attraction between macroions and coions explains the coupling between the macroion and salt densities.

The height of the first peaks in the radial distribution functions in Figs. 10 and 11 is related to the strength of the attraction. As can be seen, the macroion-macroion peak is

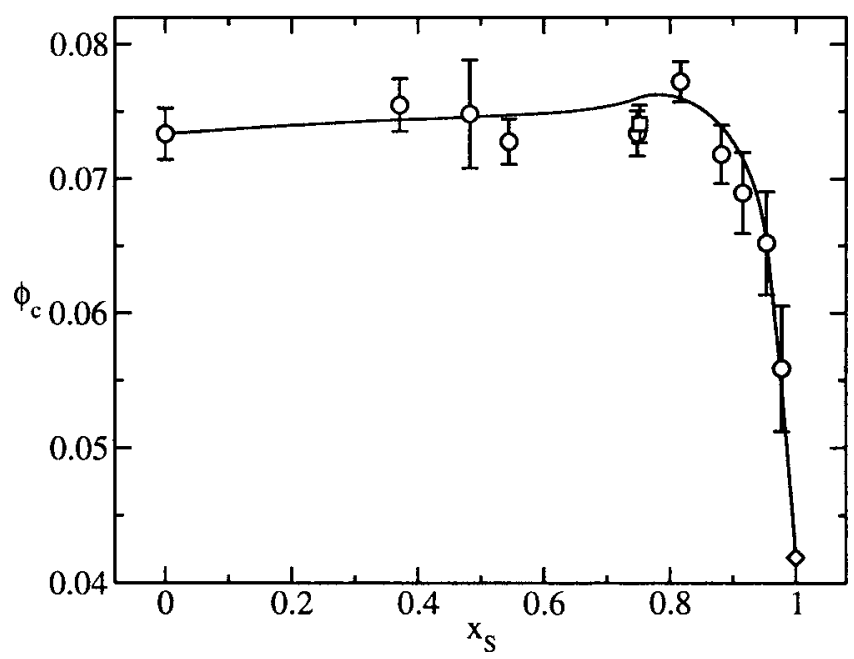

FIG. 6. Critical volume fraction $\phi_{c}\left(x_{S}\right)$ of system (b) with charge asymmetry of $3: 1$ and size asymmetry $\delta=0.667$, as a function of the salt mole fraction $x_{S}$. The points marked with the circles $(\bigcirc)$ are for box size $L$ $=12 \sigma_{M I}$ and the point marked with the square $(\square)$ is for box size $L$ $=15 \sigma_{M I}$. The line is a guide to the eye. The pure salt critical temperature at $x_{S}=1(\diamond)$ is from Ref. 27 .

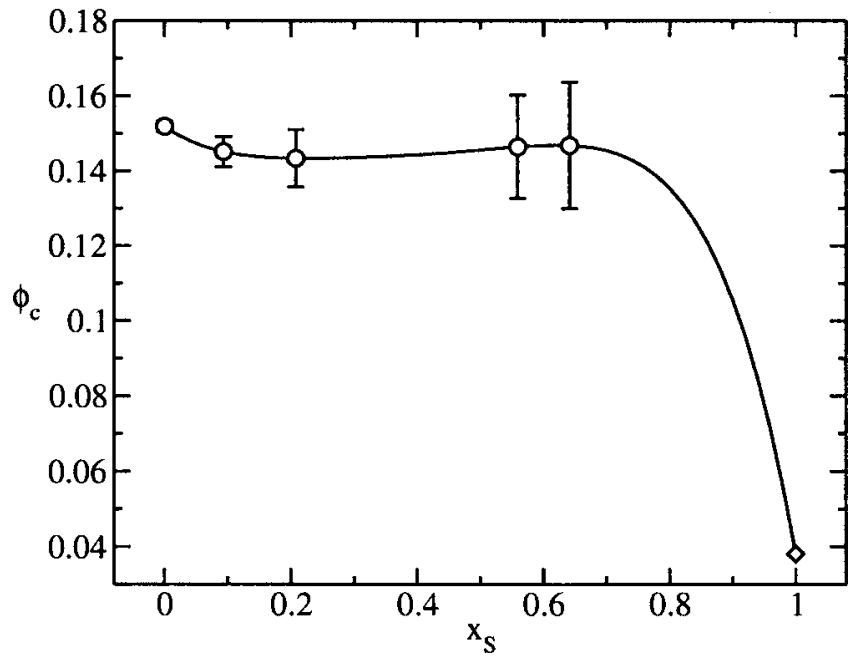

FIG. 7. Critical volume fraction $\phi_{c}\left(x_{S}\right)$ of system (c) with charge asymmetry of $10: 1$ and size asymmetry $\delta=0.9(\bigcirc)$, as a function of the salt mole fraction $x_{S}$. The results are for box size $L=12 \sigma_{M I}$. The line is a guide to the eye. The pure salt critical temperature at $x_{S}=1(\diamond)$ is from Ref. 27.

approximately twice as high for system (d) (Fig. 11) than for system (b) (Fig. 10), while the coions-coions peak is much higher for system (b) (Fig. 10) than for system (d) (Fig. 11). A simple explanation for these differences is obtained, when one considers the critical temperature in these systems relative to the salt-free $\left(x_{S}=0\right)$ and pure salt $\left(x_{S}=1\right)$ critical temperatures. Temperature in system (d) (Fig. 11) is below the critical temperature of the salt-free state, and this is why the macroion-macroion coupling is high compared to system (b) (Fig. 10), where the temperature is similar to the critical temperature of the salt-free state. Similarly, temperature in system (b) is below the critical temperature of pure salt and this is why the coions-coions coupling is high compared to system (d), where the temperature is above the critical temperature of the pure salt. The difference between the two

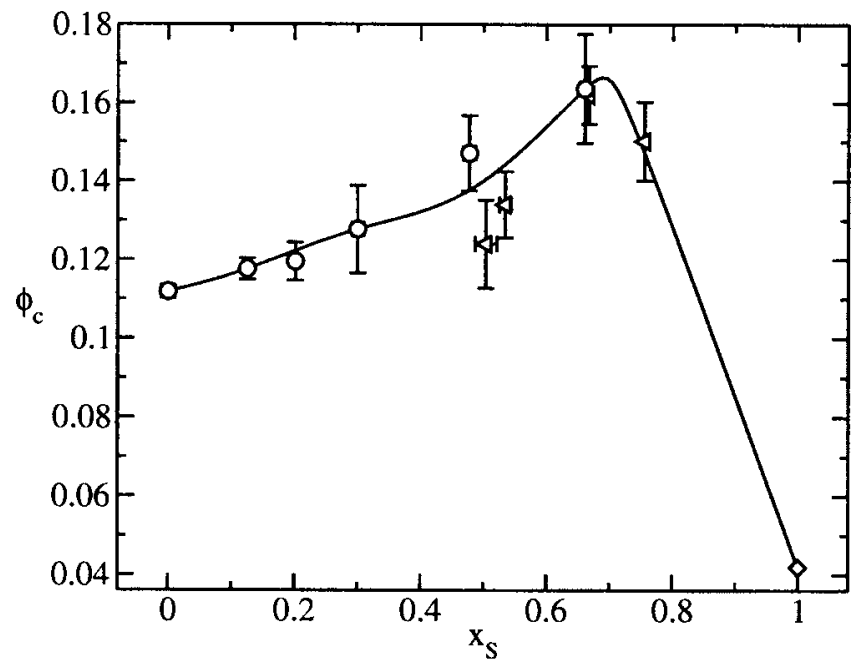

FIG. 8. Critical volume fraction $\phi_{c}\left(x_{S}\right)$ of system (d) with charge asymmetry of $10: 1$ and size asymmetry $\delta=0.667$, as a function of the salt mole fraction $x_{S}$. The points marked with the circles $(\bigcirc)$ are for box size $L$ $=12 \sigma_{M I}$ and those marked with the triangles $(\triangleleft)$ are for box size $L$ $=10 \sigma_{M I}$. The line is a guide to the eye. The pure salt critical temperature at $x_{S}=1(\diamond)$ is from Ref. 27. 


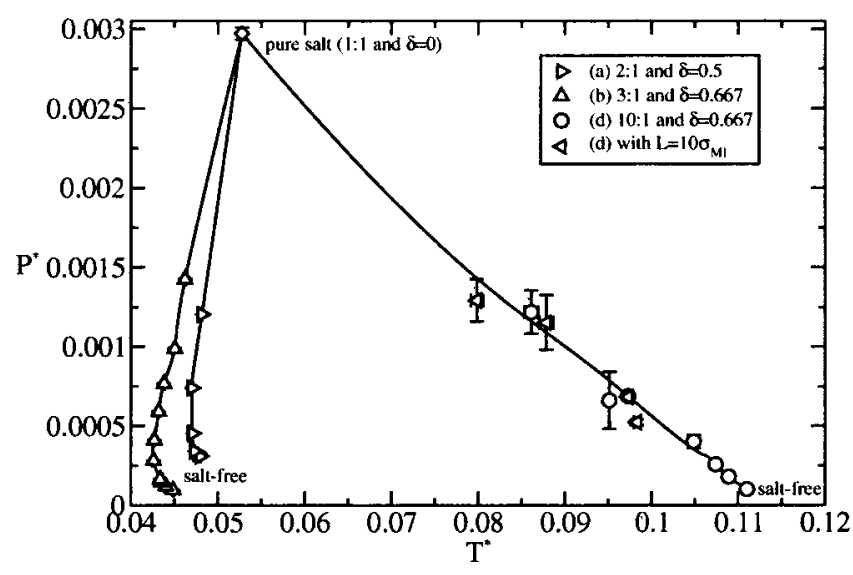

FIG. 9. Critical-point loci in the $\left(T^{*}, P^{*}\right)$ plane. The lines are guides to the eye.

system's is also well depicted in Figs. 12 and 13, where snapshots of the vapor phase close to the $x_{S}=0.48$ critical point are shown. Macroions in system (d) (Fig. 13) form rather dense clusters, while in system (b) (Fig. 12) the macroions are more loosely bound to each other. This implies that the macroion-macroion coupling is higher in system (d) than in (b). Co- and counterions in system (b) (Fig. 12) form dimers and trimers, while in system (d) (Fig. 13) there are free microions, implying that the coions-coions coupling is higher in system (b) than in (d). This agrees with the radial distribution functions in Figs. 10 and 11. Furthermore, coions in system (b) are bound close to the macroions (see Fig. 12), while in system (d) the coions fill the space left empty by the macroions (see Fig. 13). This is in agreement with the radial distribution functions in Figs. 10 and 11, which show a higher macroion-coions peak for system (b) than for (d). The clustering seen in Fig. 13 is typical for system (d) with added salt. Clusters were also seen at temperatures above the critical temperature and at densities different from the critical density.

The deepness of the macroion-macroion attraction in system (d) can be estimated from the first peak in Fig. 11 to be $\ln (10) k_{B} T \approx 2.3 k_{B} T^{35}$ This means, that each macroion gains about $2 k_{B} T$ of energy when it comes in contact with

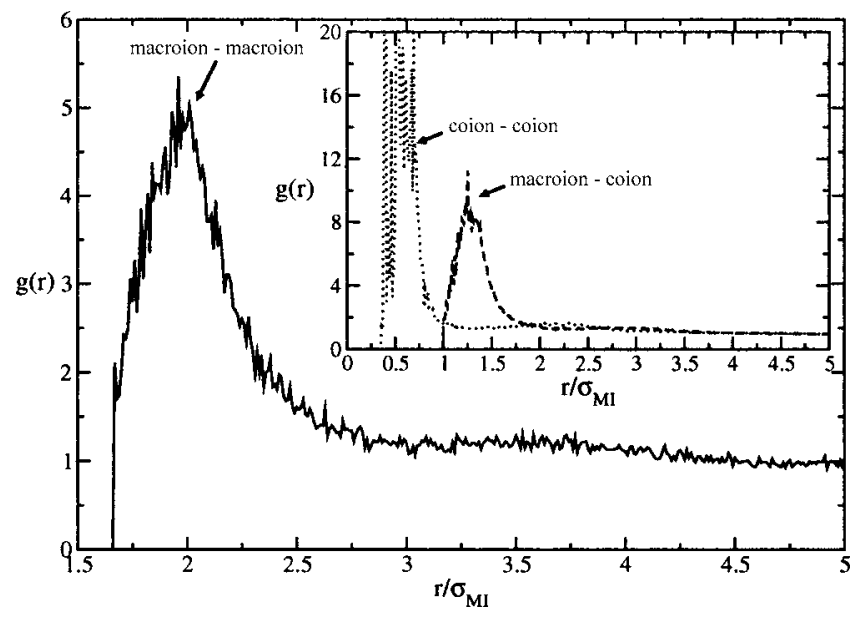

FIG. 10. Radial distribution functions for system (b) $(3: 1$ and $\delta=0.667)$ at $T^{*}=0.045 \approx 1.03 T_{c}^{*}, \mu_{S}^{*}=-1.13$, and $\phi=0.020(2)$.

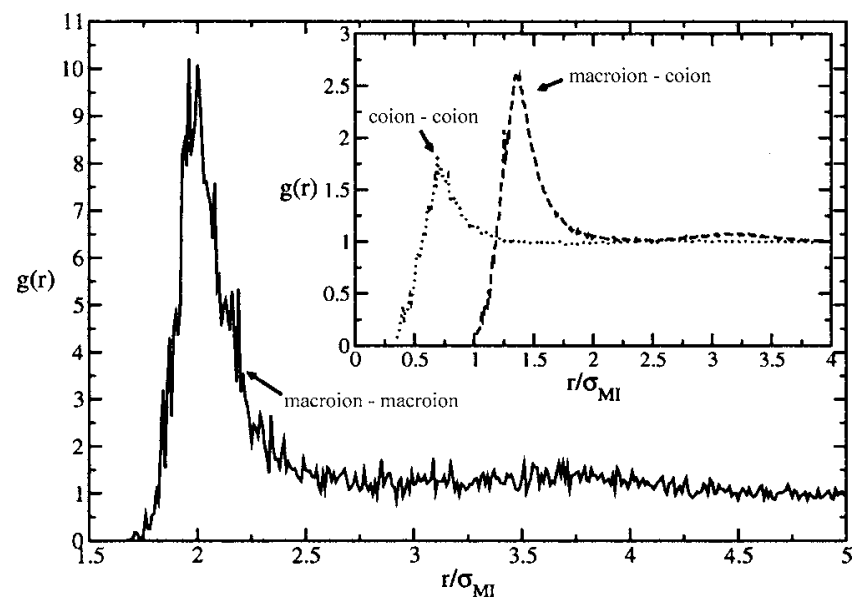

FIG. 11. Radial distribution functions for system (d) (10:1 and $\delta=0.667)$ at $T^{*}=0.1 \approx 1.03 T_{c}^{*}, \mu_{S}^{*}=-1.33$, and $\phi=0.037(2)$.

another macroion. We observed, that this energy minimum causes considerable sampling problems. Acceptance of the insertion and removal steps drops and simulations tend to get stuck in the high-density state. This is the main reason why we could not reach higher salt concentrations.

\section{CONCLUSIONS}

We have studied the critical parameters of electrolyte mixtures within the primitive model. The mixtures consist of large multivalent macroions and small monovalent co- and counterions. We view the system as a binary mixture of macroions with their counterions and salt (co- and counterion pair). We calculated the critical-point loci that connect the salt-free state consisting of macroions and counterions with the pure salt state. Critical points were calculated for four systems with charge and size asymmetries of (a) 2:1 and $\delta$ $=0.5$, (b) $3: 1$ and $\delta=0.667$, (c) $10: 1$ and $\delta=0.9$, and (d) $10: 1$ and $\delta=0.667$.

We used a combination of grand canonical Monte Carlo simulations, histogram reweighting method, and mixed-field

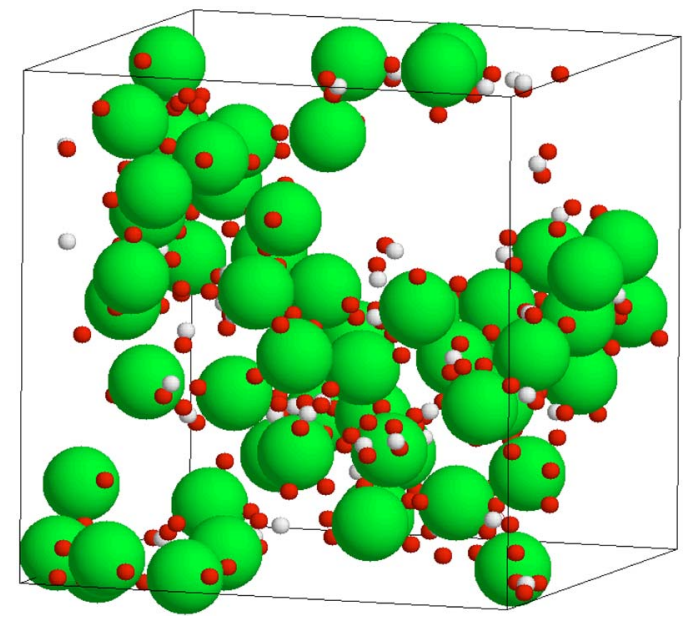

FIG. 12. (Color online) Snapshot of system (b) $(3: 1$ and $\delta=0.667)$ at the vapor phase of the $\mu_{S}^{*}=-1.13\left[x_{S}=0.482(1)\right]$ critical point with $T^{*}=0.044$ $=T_{c}^{*}, \mu^{*}=-2.730$, and $\phi=0.087$. The big spheres are macroions with charge $+3 q$, white small spheres are coions with charge $+q$, and red (dark) small spheres are counterions with charge $-q$. 


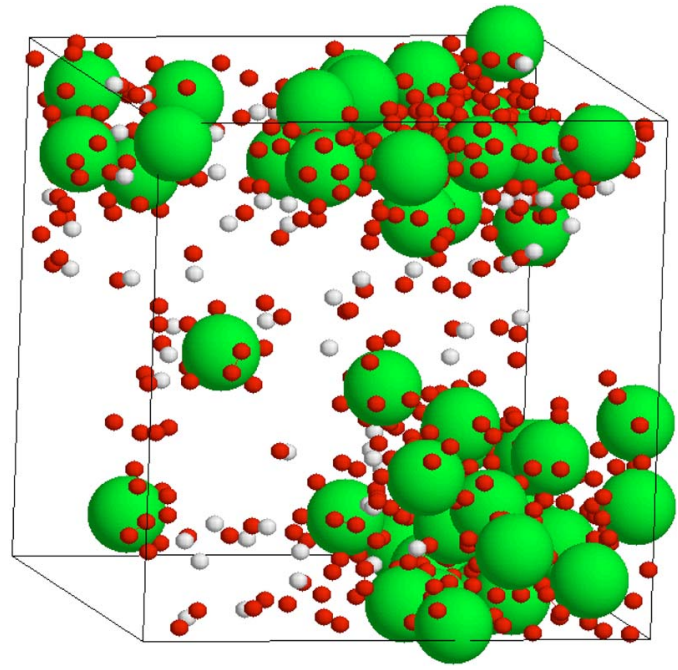

FIG. 13. (Color online) Snapshot of system (d) (10:1 and $\delta=0.667)$ close to the $\mu_{S}^{*}=-1.33\left[x_{S}=0.48(1)\right]$ critical point with $T^{*}=0.0992=1.02 T_{c}^{*}, \mu^{*}=$ -22.738 , and $\phi=0.079$. The big spheres are macroions with charge $+10 q$, white small spheres are coions with charge $+q$, and red (dark) small spheres are counterions with charge $-q$.

finite-scaling method to obtain the critical points. The simulations were done using fine-lattice discretization method. We implemented a distance biasing method that enhances the efficiency of the grand canonical insertion and removal steps by many orders of magnitude and enables direct simulations of systems with charge asymmetry of 10:1. In systems (c) and (d), at high salt (coions) concentration, we experienced sampling problems that inhibited us from calculating critical points closer to the pure salt limit.

We observed the following nonmonotonic behavior in the critical parameters: (i) in systems (a)-(c), the critical temperature $T_{c}^{*}$ as a function of the salt mole fraction $x_{S}$ goes through a minimum, and (ii) in system (d), the critical volume fraction $\phi_{c}\left(x_{S}\right)$ goes through a maximum. The binary electrolyte mixtures studied in this paper are type-I mixtures, where the two species mix continuously.

As the salt has nonmonotonic effects on the critical parameters of electrolytes, our results should form a good benchmark for testing theoretical models on electrolytes. In particular, it would be interesting to see if a theory can reproduce the nonmonotonic behavior of $T_{c}^{*}\left(x_{S}\right)$ and $\phi_{c}^{*}\left(x_{S}\right)$, and predict the type of the mixture correctly.

For future studies, an idea that could help in calculating critical points close to the pure salt limit is to reduce the system size at high salt concentrations. Smaller system size means shorter simulation time and, if done correctly, should not introduce considerable finite-size effects because the overall density of the system decreases in the pure salt limit.

\section{ACKNOWLEDGMENTS}

We would like to thank Vladimir Lobaskin and Daniel Cheong for useful discussions. At Utrecht, this work is part of the research program of the "Stichting voor Fundamenteel Onderzoek der Materie (FOM)," which is financially supported by the "Nederlandse Organisatie voor Wetenschappelijk Onderzoek (NWO)." At Princeton, this work has been supported by the Department of Energy, Office of Basic Energy Sciences (Grant No. DE-FG201ER15121), with additional support provided by ACS-PRF (Grant No. 3815-AC9).

${ }^{1}$ M. Kleemeier, S. Wiegand, W. Schröer, and H. Weingärtner, J. Chem. Phys. 110, 3085 (1999).

${ }^{2}$ H. L. Bianchi and M. L. Japas, J. Chem. Phys. 115, 10472 (2001).

${ }^{3}$ M. E. Fisher and Y. Levin, Phys. Rev. Lett. 71, 3826 (1993).

${ }^{4}$ G. Stell, J. Stat. Phys. 78, 197 (1995).

${ }^{5}$ Y. Levin and M. E. Fisher, Physica A 225, 164 (1996).

${ }^{6}$ E. González-Tovar, Mol. Phys. 97, 1203 (1999).

${ }^{7}$ D. M. Zuckerman, M. E. Fisher, and S. Bekiranov, Phys. Rev. E 64, 011206 (2001).

${ }^{8}$ M. N. Artyomov, V. Kobelev, and A. B. Kolomeisky, J. Chem. Phys. 118, 6394 (2003).

${ }^{9}$ J. M. Romero-Enrique, G. Orkoulas, A. Z. Panagiotopoulos, and M. E. Fisher, Phys. Rev. Lett. 85, 4558 (2000).

${ }^{10}$ A. Z. Panagiotopoulos and M. E. Fisher, Phys. Rev. Lett. 88, 045701 (2002).

${ }^{11}$ E. Luijten, M. E. Fisher, and A. Z. Panagiotopoulos, Phys. Rev. Lett. 88, 185701 (2002).

${ }^{12}$ D. Cheong and A. Z. Panagiotopoulos, J. Chem. Phys. 119, 8526 (2003).

${ }^{13}$ Y. C. Kim and M. E. Fisher, Phys. Rev. Lett. 92, 185703 (2004).

${ }^{14}$ Q. L. Yan and J. J. de Pablo, J. Chem. Phys. 111, 9509 (1999).

${ }^{15}$ Q. L. Yan and J. J. de Pablo, J. Chem. Phys. 114, 1727 (2001).

${ }^{16}$ Q. L. Yan and J. J. de Pablo Phys. Rev. Lett. 86, 2054 (2001).

${ }^{17}$ J. Reščič and P. Linse, J. Chem. Phys. 114, 10131 (2001).

${ }^{18}$ P. Linse and V. Lobaskin, J. Chem. Phys. 112, 3917 (2000).

${ }^{19}$ V. Lobaskin and K. Qamhieh, J. Phys. Chem. B 107, 8022 (2003).

${ }^{20}$ Y. C. Kim and M. E. Fisher, J. Phys. Chem. B 105, 11785 (2001).

${ }^{21}$ D. G. Angelescu and P. Linse, Langmuir 19, 9661 (2003).

${ }^{22}$ R. Zhang and B. I. Shklovskii, Phys. Rev. E 69, 021909 (2004).

${ }^{23}$ G. Orkoulas and A. Z. Panagiotopoulos, J. Chem. Phys. 101, 1452 (1994).

${ }^{24}$ F. H. Stillinger and R. Lovett, J. Chem. Phys. 48, 3858 (1968).

${ }^{25}$ D. Frenkel and B. Smit, Understanding Molecular Simulations, 2nd ed. (Academic, New York, 2002).

${ }^{26}$ P. P. Ewald, Ann. Phys. 64, 253 (1921).

${ }^{27}$ A. Z. Panagiotopoulos and S. K. Kumar, Phys. Rev. Lett. 83, 2981 (1999).

${ }^{28}$ A. Z. Panagiotopoulos, J. Chem. Phys. 116, 3007 (2002).

${ }^{29}$ N. B. Wilding and A. D. Bruce, J. Phys.: Condens. Matter 4, 3087 (1992).

${ }^{30}$ Y. C. Kim and M. E. Fisher, J. Phys. Chem. B 108, 6750 (2004).

${ }^{31}$ A. M. Ferrenberg and R. H. Swendsen, Phys. Rev. Lett. 61, 2635 (1988).

${ }^{32}$ A. M. Ferrenberg and R. H. Swendsen, Phys. Rev. Lett. 63, 1195 (1989).

${ }^{33}$ R. Evans, U. Marini Bettolo Marconi, and P. Tarazona, J. Chem. Phys. 84, 2376 (1986).

${ }^{34}$ J. S. Rowlinson and F. L. Swinton, Liquids and Liquid Mixtures, 3rd ed. (Butterworth, London, 1982).

${ }^{35}$ Here, the validity of the approximation $u_{\mathrm{eff}}=-\ln g(r)$ was checked by calculating the radial distribution at lower densities. At $\phi \approx 0.00479(2)$ the depth of the attraction was estimated to be $\ln (16) k_{B} T \approx 2.8 k_{B} T$. 